\title{
The evolution of the graphic-symbolic communication positioning of the technological brand
}

\author{
Keywords \\ Advertising, Brand positioning, Semiotics, Technology, Visual communication.
}

With the globalization process, the concept of technology is widespread in the construction of a more simplified society (Harvey, 2008). Over time, technology evolves and transforms, during this process, communication and culture follow the changes. Companies inserted in this sociocultural network seek to communicate with the consumer, and thus the positioning is irrefutable in this marketing process. If technology, communication, and culture change and transform over time, then brand positioning must also follow this movement (Sant'Anna, 1998). Advertising uses the word technology as a sales argument and to position the brand in the minds of consumers, however, there is confusion in the representation of graphic visuality in technology companies when it comes to giving imagery meaning to the technology itself. It is believed that part of it is given by the cultural, political, and tooling aspects available for these constructions, as well as, it is intended to study the possible cause of hypertrophy of the aesthetic function in communication products, a concept addressed by Perassi (2001) when elucidating a pathology of representations that mischaracterize the referential and advertising function. Based on this context, the theme of this proposal will describe the evolution process of the graphicsymbolic communication positioning of the Multilaser technological brand, and thus analyze the changes in positioning from the perspective of design. The chosen brand brings relevance to the study because it went through historical milestones in its business and communication structure, which will contribute to the descriptive analysis of its evolution. The company was founded in Brazil in 1987.
It started operating in the printer and photocopying segment, recycling cartridges until 2003, after the company entered the computer, accessories, and cell phone line, and consequently changed its positioning and starts to compete with major players in the world technological market. It currently has 44 thousand points of sale in Brazil, and portfolio of 15 departments. The problem of the proposal takes into account a large amount of importation of technological products, and soon it is believed in the importation of communication, and design references. The study is justified by the need to assess the perception of confusion in graphic representation and hypertrophy of the aesthetic function in communication in the Brazilian technological segment. To achieve the result, a qualitative methodological approach will be used, aimed at the perception of meanings that are intrinsic in beliefs, values, and attitudes in human relationships. Therefore, so that the research objectives are achieved, the study will be divided into three stages of development: exploratory, bibliographical, and documentary. The discussions that take place in this proposal bring the relationship between communication and culture, visual communication as a form of brand positioning, as well as graphic advertising representation in communication from the perspective of design. The study will be limited to graphic products for advertising communication, which is expected to understand a look at the advancement of knowledge in visuality and graphic-symbolic positioning of Brazilian technology brands, as well as the role of professionals in the field of design. 